\section{e0500 ASSOCIATION BETWEEN RED BLOOD CELL DISTRIBUTION WIDTH (RDW) AND ALL-CAUSE MORTALITY IN PATIENTS UNDERGOING PERCUTANEOUS CORONARY INTERVENTION}

doi:10.1136/hrt.2010.208967.500

Han Hongya, Zhou Yujie, Ma Hanying, Liu Yuyang, Shi Dongmei, Zhao Yingxin, Liu Xiaoli, Gao Fei, Shen Hua. Beijing Anzhen Hospital

Background Red blood cell distribution width (RDW) has been shown to be an independent predictor of mortality in patients with coronary artery disease and in patients with heart failure. However, there are limited clinical studies about the prognostic value of RDW in patients with coronary artery disease (CAD) undergoing percutaneous coronary intervention (PCI). We aimed to examine the association between RDW on admission and the risk of all-cause mortality in patients with CAD undergoing PCI.

Methods We analysed RDW values on admission in 800 consecutively adult patients, who were admitted to our hospital undergoing PCI for $\mathrm{CAD}$. In all patients, a baseline blood sample was collected for routine haematological testing, at the same time the plasma level of highsensitivity C-reactive protein (hsCRP), erythrocyte sedimentation rate (ESR) and B-type natriuretic peptide (BNP) were tested as well; patients who were anaemic at baseline were excluded. All patients were followed prospectively for all-cause mortality.

Results After a median follow up of five (IR 4.6-5.6) years, there were a total of $48(6 \%)$ deaths. RDW was analysed as a categorical variable with empirically determined cut points of 13.2 and 14.5 (low RDW $<13.2$, medium RDW $>/=13.2$ to $<14.5$, high RDW $>/=14.5$ ) based on differences in HR for death among RDW deciles. In univariate analysis, higher RDW was a significant predictor of mortality $(p<0.01)$, HR for death in patients with high RDW relative to low RDW was 5.1 (95\% CI (CI): 2.0 to 13.0). In the high RDW group, the values of hsCRP, ESR and BNP were higher than that in the low or medium group $(\mathrm{p}<0.05)$.

Conclusions As RDW is widely available to clinicians as a part of the complete blood cells count, and therefore incurs no additional costs. Higher RDW might be a strong and independent predictor of longterm mortality in patients undergoing PCI who were not anaemic at baseline. Neurohumoral activation may be a mechanistic link between increased RDW and adverse events in patients with $\mathrm{CAD}$ undergoing PCI.

\section{e0501 1-YEAR CLINICAL OUTCOMES AFTER IMPLANTATION OF SIROLIMUS-ELUTING STENTS IN PATIENTS WITH CORONARY ARTERY DISEASE AND TYPE 2 DIABETES}

doi:10.1136/hrt.2010.208967.501

Han Hongya, Zhou Yujie, Ma Hanying, Liu Yuyang, Shi Dongmei, Zhao Yingxin, Liu Xiaoli, Gao Fei, Shen Hua. Beijing Anzhen Hospital

Background Drug-eluting stents (DES) have been shown to significantly reduce clinical events and angiographic restenosis in the treatment of coronary artery disease (CAD). We aimed to assess the long-term efficacy and safety of two Sirolimus-Eluting Stents (the biodegradable polymer-coated sirolimus-eluting stents, EXCEL and the polymer-based sirolimus-eluting cobalt-chromium stents, Firebird 2) in the treatment of patients with $\mathrm{CAD}$ and Type 2 Diabetes.

Methods A total of 320 consecutive patients with coronary artery disease and type 2 diabetes undergoing percutaneous coronary intervention randomised into two groups (EXCEL group, $n=158$; Firebird 2 group, $n=162$ ). Baseline clinical characteristics, procedural success rate and occurrence of major adverse cardiac events (MACE, a composite of death, myocardial infarction or target lesion revas- cularisation) during follow-up were recorded and compared between the two groups.

Results During hospitalisation no patient had complications. At 1 year follow-up, both of the EXCEL group and Firebird 2 group had lower occurrence of cardiac events $(1.9 \%$ in the Excel group and $1.2 \%$ in the Firebird group, $\mathrm{p}>0.05)$.

Conclusion Implantation of EXCEL stent or Firebird 2 stent for patients with coronary artery disease and type 2 diabetes is safe and can reduce major adverse cardiac events in long-term follow-up.

\section{e0502 RELATIONSHIP BETWEEN ACTIVATED CLOTTING TIME AND THE CLINICAL OUTCOMES AFTER TRANSRADIAL CORONARY STENTING}

doi:10.1136/hrt.2010.208967.502

Han Hongya, Zhou Yujie, Ma Hanying, Yang Shiwei, Jia Dean, Wang Zhijian, Nie Bin, Liu Xiaoli, Gao Fei, Shen Hua. Beijing Anzhen Hospital

Background Despite significant pharmacological and mechanical advancements in PCI, The optimal value of activated clotting time (ACT) during percutaneous coronary intervention (PCI) with unfractionated heparin remains controversial. No data are available on the relation between the ACT at the end of the procedure (final ACT) and the clinical outcomes after transradial PCI.

Methods A total of 682 consecutive patients with acute coronary syndrome after transradial Stenting were enrolled into our study, final ACT was available in 658 (96\%). All patients were pretreated with aspirin and clopidogrel. After radial sheath insertion, patients received $70 \mathrm{IU} / \mathrm{kg}$ unfractionated heparin. Baseline clinical characteristics, procedural success rate, major bleeding and occurrence of major adverse cardiac events (MACE, a composite of death, myocardial infarction or target lesion revascularisation) during 30 days and 1 year follow-up were recorded.

Results The median final ACT value was $312 \mathrm{~s}$ (IR 262 to 352). At 30 days, the rate of MACE, from the lower to upper groups was $2.7 \%, 4.0 \%$, and $2.0 \%$, respectively ( $p>0.05)$, and the rate of major bleeding was $1.2 \%, 0.9 \%$ and $0.6 \%$, respectively ( $p>0.05$ ). During the 1 year of follow-up, the incidence of myocardial infarction was less with the greatest ACT value (>320 s) than in the other 2 groups $(4.0 \%, 7.9 \%$, and $7.6 \%$, respectively; p320 seconds remained associated with a $42 \%$ relative reduction in myocardial infarction (OR $0.51,95 \%$ CI 0.24 to $0.89, \mathrm{p}<0.05$ ). The rate of major bleeding was $0.9 \%, 0.6 \%$ and $0.3 \%$, respectively ( $p>0.05$ ). Death and target vessel revascularisation remained similar in all groups for 320 seconds appears protective after transradial coronary stenting, and this benefit was maintained for $<$ or $=1$ year. With a transradial approach and antiplatelet therapy, greater ACT values did not correlate with an increased risk of bleeding.

\section{E0503 IMPACT OF RENAL FUNCTION ON OUTCOMES OF PATIENTS UNDERGOING COMPLETE REVASCULARISATION FOR THE TREATMENT OF MULTIVESSEL CORONARY ARTERY DISEASE}

doi:10.1136/hrt.2010.208967.503

Wang Zhijian, Zhou Yujie, Zhao Yinxin, Shi Dongmei, Liu Yuyang, Gao Fei. Anzhen Hospital Capital Medical University, Beijing, China

Background Chronic kidney disease (CKD) is a strong predictor of adverse cardiac events after revascularisation for patients with multiple coronary artery disease. However, the comparison of the two strategies of revascularisation, percutaneous coronary intervention (PCI) or coronary bypass grafting surgery (CABG), in this context is limited. 